\title{
ON UNIONS OF CHAINS OF MODELS
}

\author{
C. C. CHANG
}

The main purpose of this note is to prove the following theorem in the theory of models: Let $K$ be an arithmetical class (in the wider sense) of relational systems. Then $K$ is the class of all models of some finite or infinite set of sentences of the form

$$
\Pi x_{1}, \cdots, x_{n} \Sigma y_{1}, \cdots, y_{m} \nu
$$

where $\nu$ is quantifier-free, if and only if $K$ is closed under unions of chains of models. ${ }^{1}$ We have adopted most of the notational features of Tarski [7], in which can be found the definitions of relational systems, subsystems, and unions of a chain of systems; we have assumed familiarity with the symbolism of [7]. We have also adopted the convention of giving definitions and theorems only for a similarity class of systems of the type $\langle A, S\rangle$ where $S$ is a ternary relation over $A$. All the results we have obtained here can be carried over trivially for similarity classes of systems in which there are a finite number of finitary relations.

It will be apparent to the reader that this paper exploits a special method and a special theorem. The method is that of graph-diagrams first introduced in Robinson [6], and the theorem is the completeness theorem of Gödel [3]. We shall use the completeness theorem in the generalized form (by Malcev and Henkin) where we allow an arbitrary number of constants in the first-order predicate calculus. The plan of the paper is first to give a mathematical characterization of those classes $K$ which are determined by sentences of the form $(*)$, then to use this characterization to prove our main result. ${ }^{2}$ At the end, we shall apply our result to convex arithmetical classes.

Presented to the Society August 24, 1956; received by the editors February 20, 1958.

, 1 This result was announced by the author in [2]. It has previously been announced by $Ł o s$ and Suszko in [4] and their joint result recently appeared in [5]. It seems that the two methods of proof are entirely different, and our results (Theorem 7 and Theorem 11) are stronger results than those in [5]. The author takes this opportunity to thank A. Tarski and R. Grewe for pointing out some simplifications in the proofs of Lemmas 8,9 , and 10 . These simplifications utilize some results of Tarski and Vaught [9] which were not known to the author.

2 The characterizations given in Theorems 4, 6, and 7 bear a close analogy to the corresponding characterizations given by Tarski in [7] for universal arithmetical classes. 
Definition 1. A class $K$ of relational systems $\langle A, S\rangle$ is an universal-existential class [in the wider sense], in symbols $K \in U E C\left[K \in U E C_{\Delta}\right]$, if and only if $K$ is the class of all models of a' first-order sentence [a set of first-order sentences] of the form $(*)$.

Let relational systems $\mathfrak{A}=\langle A, S\rangle, \mathfrak{A}^{\prime}=\left\langle A^{\prime}, S^{\prime}\right\rangle$, and $\mathfrak{B}=\langle B, T\rangle$ be given where $\mathfrak{X}^{\prime} \in S(\mathfrak{U})$, that is, $\mathfrak{U}^{\prime}$ is a subsystem of $\mathfrak{A}$, and let $m$ be an arbitrary natural number.

Definition 2. $\mathfrak{B}$ is an $m$-cover of $\mathfrak{A}$ with respect to $\mathfrak{A}^{\prime}$ if, and only if,

(i) $\mathfrak{A}^{\prime}$ is isomorphically embeddable in $\mathfrak{B}$ by an isomorphism $f$, and

(ii) every subsystem $\mathfrak{B}^{\prime}=\left\langle B^{\prime}, T^{\prime}\right\rangle$ of $\mathfrak{B}$ where $f^{*}\left(A^{\prime}\right) \subseteq B^{\prime}$, and where the set difference $B^{\prime}-f^{*}\left(A^{\prime}\right)$ contains no more than $m$ elements, can be isomorphically embedded in $\mathfrak{A}$ by an isomorphism $g$ where $g^{-1}$ restricted to the set $A^{\prime}$ is equal to $f$.

Let $\mathfrak{A}^{\prime}=\left\langle A^{\prime}, S^{\prime}\right\rangle$ be a finite subsystem of $\mathfrak{A}=\langle A, S\rangle$, and let $A^{\prime}=\left\{a_{1}, \cdots, a_{n}\right\}$. Consider all possible extensions of $A^{\prime}$ in $A$ with no more than $n+m$ elements. Each such extension $C$ will give rise to a subsystem $\mathfrak{E}=\left\langle C, S \cap C^{3}\right\rangle$ of $\mathfrak{A}$. Since $S$ is a ternary relation (or, since there are only a finite number of finitary relations), there will exist among all such extensions a finite number of subsystems $\mathfrak{\complement}_{1}, \cdots, \mathfrak{E}_{r}$ with the property that, for any extension $\mathbb{S}$ of $\mathfrak{A}^{\prime}$ with no more than $n+m$ elements, there exists a $\mathfrak{S}_{i}, 1 \leqq i \leqq r$, such that $\mathfrak{S} \cong \mathfrak{S}_{i}$ and where the isomorphism is the identity on the set $A^{\prime}$. Let $C_{i}$ be the set of elements $\left\{a_{1}, \cdots, a_{n}, b_{1}, \cdots, b_{m}\right\}$ where the $b_{j}$ 's need not be distinct for distinct indices. We introduce a constant $c_{a}$ for each element $a \in C_{i}$, and we define the formula $\nu_{i}\left(c_{a_{1}}, \cdots, c_{a_{n}}, c_{b_{1}}, \cdots, c_{b_{m}}\right)$ as follows: Let $x_{1}, x_{2}, x_{3}$ be elements of $C_{i}$. We write

$$
\begin{aligned}
S\left(c_{x_{1}}, c_{x_{2}}, c_{x_{3}}\right) & \text { if }\left\langle x_{1}, x_{2}, x_{3}\right\rangle \in S, \\
\sim S\left(c_{x_{1}}, c_{x_{2}}, c_{x_{3}}\right) & \text { if }\left\langle x_{1}, x_{2}, x_{3}\right\rangle \in S, \\
c_{x_{1}} \equiv c_{x_{2}} & \text { if } \quad x_{1}=x_{2}, \\
c_{x_{1}} \neq c_{x_{2}} & \text { if } \quad x_{1} \neq x_{2} .
\end{aligned}
$$

The set of all formulas listed on the left constitutes a description of $\mathfrak{F}_{i}$. Since $\mathfrak{S}_{i}$ is finite, this set of formulas is clearly finite, and we let the formula $\nu_{i}\left(c_{a_{1}}, \cdots, c_{a_{n}}, c_{b_{1}}, \cdots, c_{b_{m}}\right)$ be the conjunction of all formulas in the description of $\mathfrak{C}_{i}$. Having defined the formulas $\nu_{i}$, a formula $\nu$ depending on the system $\mathfrak{A}$, the subsystem $\mathfrak{U}^{\prime}$, and the number $m$ is defined by the equality

$$
(* *) \begin{array}{r}
\nu\left(c_{a_{1}}, \cdots, c_{a_{n}}\right)=\Pi y_{1}, \cdots, y_{m}\left\{\nu_{1}\left(c_{a_{1}}, \cdots, c_{a_{n}}, y_{1}, \cdots, y_{m}\right)\right. \\
\left.\vee \cdots \vee v_{r}\left(c_{a_{1}}, \cdots, c_{a_{n}}, y_{1}, \cdots, y_{m}\right)\right\} .
\end{array}
$$


Finally, we define $\xi=\Sigma x_{1}, \cdots, x_{n} \nu\left(x_{1}, \cdots, x_{n}\right)$. The notation introduced in this paragraph will be used in the proofs of Lemma 3, Theorem 6, and Theorem 11.

Lemma 3. Let $\mathfrak{A}$ be a finite subsystem of $\mathfrak{A}$ and let $K$ be the class of all systems $\mathfrak{B}$ where $\mathfrak{B}$ is not an m-cover of $\mathfrak{A}$ with respect to $\mathfrak{A}^{\prime}$. Then there exists an $L \in U E C$ such that $K \subseteq L$ and $\mathfrak{P} \notin L$.

Proof. Let $L$ be the class of all models of the sentence $\xi$ introduced above. Clearly, $L \in U E C, K \subseteq L$, and $\mathfrak{A} \notin L$.

THEOREM 4. The following two conditions are equivalent:

(i) $K \in U E C_{\Delta}$.

(ii) If for each finite subsystem $\mathfrak{A}^{\prime}$ of $\mathfrak{A}$ and each $m$ there exists a system $\mathfrak{B} \in K$ where $\mathfrak{B}$ is an m-cover of $\mathfrak{A}$ with respect to $\mathfrak{X}^{\prime}$, then $\mathfrak{A} \in K$.

Proof. Assume (i). Let $\mathfrak{A}=\langle A, S\rangle$ satisfy the hypothesis of (ii) and let $\xi=\Pi x_{1}, \cdots, x_{n} \Sigma y_{1}, \cdots, y_{m} \nu\left(x_{1}, \cdots, x_{n}, y_{1}, \cdots, y_{m}\right)$ be any one of the characterizing sentences of $K$. We wish to show that $\xi$ holds in $\mathfrak{A}$. Let any $n$ elements of $A$ be chosen, let $A^{\prime}=\left\{a_{1}, \cdots, a_{n}\right\}$ be the set of those elements, and let $\mathfrak{A}^{\prime}=\left\langle A^{\prime}, S^{\prime}\right\rangle$ be the subsystem determined by $A^{\prime}$. Let $\mathfrak{B}=\langle B, T\rangle$ be an $m$-cover of $\mathfrak{A}$ with respect to $\mathfrak{A}^{\prime}$ and let $\left\{f\left(a_{1}\right), \cdots, f\left(a_{n}\right)\right\} \subseteq B$ be the image of $A^{\prime}$ under the isomorphism $f$ given by $2(\mathrm{i})$. Since $\mathfrak{B} \in K$, there exist elements $b_{1}, \cdots, b_{m} \in B$ for which

$$
\nu\left(c_{f\left(a_{1}\right)}, \cdots, c_{f\left(a_{n}\right)}, c_{b_{1}}, \cdots, c_{b_{m}}\right)
$$

holds in $\mathfrak{B}$ under the interpretation of $b$ for $c_{b}$ for all $b \in B$. Applying now the isomorphism $g$ given by 2 (ii), there exist elements $g\left(b_{1}\right), \cdots$, $g\left(b_{m}\right) \in A$ for which

$$
\nu\left(c_{a_{1}}, \cdots, c_{a_{n}}, c_{g\left(b_{1}\right)}, \cdots, c_{g\left(b_{m}\right)}\right)
$$

holds in $\mathfrak{A}$ under the interpretation of $a$ for $c_{a}$ for all $a \in A$. Since the choice of the elements $a_{1}, \cdots, a_{n}$ is arbitrary, we see that $\xi$ holds in $\mathfrak{A}$. Hence, $\mathfrak{A} \in K$.

On the other hand, assume that $K$ satisfies 4 (ii). Let

$$
M=\cap\{L ; L \in U E C \text { and } K \subseteq L\} .
$$

Clearly $K \subseteq M$ and $M \in U E C_{\Delta}$. We wish to show the equality $K=M$. Assume $\mathfrak{A} \notin K$, thus, by 4 (ii), there exists a finite subsystem $\mathfrak{A}^{\prime}$ of $\mathfrak{A}$ and an $m$ for which no $\mathfrak{B} \in K$ is an $m$-cover of $\mathfrak{A}$ with respect to $\mathfrak{A}^{\prime}$. Obviously, $K$ is included in the class $L$ defined in Lemma 3 and $\mathfrak{A} \notin L$, thus $\mathfrak{A} \in M$. This proves the inclusion $M \subseteq K$ and hence the equality $K=M$. 
We now introduce a notion which is stronger than the notion of a subsystem.

Definition 5. $\mathfrak{A} \in S^{*}(\mathfrak{B})$ if, and only if, $\mathfrak{A} \in S(\mathfrak{B})$ and every finite subsystem $\mathfrak{B}^{\prime}$ of $\mathfrak{B}$ can be mapped isomorphically onto a finite subsystem $\mathfrak{U}^{\prime}$ of $\mathfrak{A}$ by an isomorphism $f$ which is the identity mapping on the set $B^{\prime} \cap A$.

For a class $K$, we let $S^{*}(K)=\{\mathfrak{A}$; there exists $\mathfrak{B} \in K$ such that $\left.\mathfrak{A} \in S^{*}(\mathfrak{B})\right\}$.

The following theorem is an analog of a result of Tarski [7] on universal arithmetical classes.

THEOREM 6. The following two conditions are equivalent:

(i) $K \in U E C_{\Delta}$.

(ii) $K \in A C_{\Delta}$ and $S^{*}(K) \subseteq K$.

Proof. By an argument similar to the proof of 4(ii) from 4(i) we easily obtain 6(ii) from 6(i). Assume now 6(ii). Let $\mathfrak{A}=\langle A, S\rangle$ satisfy the hypothesis of 4 (ii), and let $\Sigma$ be the set of sentences characterizing $K$. Consider the following enlarged set of sentences $\Sigma^{\prime}$ consisting of:

(1) the set $\Sigma$,

(2) the set of formulas which forms the description of $\mathfrak{A}$ using the constants $c_{a}$ for each $a \in A$, and

(3) for each finite subset $\left\{a_{1}, \cdots, a_{n}\right\}$ of $A$ and each natural number $m$, the sentence $\nu\left(c_{a_{1}}, \cdots, c_{a_{n}}\right)$ introduced in $(* *)$.

We assert that the set $\Sigma^{\prime}$ has a model. Any finite subset $\Sigma^{\prime \prime}$ of $\Sigma^{\prime}$ will involve at most a finite number of constants $c_{a_{1}}, \cdots, c_{a_{n}}$ and a maximal natural number $m$. Since $\mathfrak{A}$ satisfies the hypothesis of 4 (ii), we let $\mathfrak{B} \in K$ be an $m$-cover of $\mathfrak{A}$ with respect to the system generated by the set $\left\{a_{1}, \cdots, a_{n}\right\}$. Obviously $\mathfrak{B}$ is a model for $\Sigma^{\prime \prime}$. Hence, by the compactness theorem, there exists a model $\mathbb{S}$ for $\Sigma^{\prime}$. From the contents of the set $\Sigma^{\prime}$, we see that $\mathfrak{S} \in K$ and that $\mathfrak{A}$ is isomorphic to a system $\mathfrak{A}^{\prime}$ where $\mathfrak{A}^{\prime} \in S^{*}(\mathfrak{E})$. By $6(\mathrm{ii}), \mathfrak{A}^{\prime} \in K$ and, since $K$ is closed under taking isomorphic models, $\mathfrak{A} \in K$. Thus 6(ii) leads to 4(ii), and by Theorem 4 , we obtain $6(\mathrm{i})$.

Just like its analog for universal classes, Theorem 6 can be improved to

Theorem 7. If $K \in A C_{\Delta}$, then $S^{*}(K) \in U E C_{\Delta}$.

Proof. We merely have to show that $S^{*}(K)$ satisfies condition 4(ii). Suppose $\mathfrak{A}$ is such that for each finite subsystem $\mathfrak{A}^{\prime}$ of $\mathfrak{A}$ and each $m$ there exists a $\mathfrak{B} \in S^{*}(K)$ such that $\mathfrak{B}$ is an $m$-cover of $\mathfrak{A}$ with respect to $\mathfrak{Y}^{\prime}$. Notice that since $\mathfrak{B} \in S^{*}(K)$, there exists a $\mathfrak{B}^{\prime} \in K$ such that $\mathfrak{B} \in S^{*}\left(\mathfrak{B}^{\prime}\right)$. It follows easily from Definitions 2 and 5 that $\mathfrak{B}^{\prime}$ 
is also an $m$-cover of $\mathfrak{A}$ with respect to $\mathfrak{A}^{\prime}$. By the device used in Theorem 6, there exist models $\mathbb{E}$ and $\mathfrak{D}$ such that $\mathfrak{S} \in K, \mathfrak{D} \in S^{*}(\mathfrak{E})$, and $\mathfrak{A}$ isomorphic to $\mathfrak{D}$. Since $K$ is closed under taking isomorphic models, we conclude that $\mathfrak{A} \in S^{*}(K)$.

At this point we pause to mention that the following result in the theory of models is implied by Theorem 7, namely: If $K \in A C_{\Delta}$ and every model of $K$ is finite, then $K \in U E C_{\Delta} \cdot{ }^{3}$ Clearly, under the hypothesis that every model of $K$ is finite, $S^{*}(K)=K$ thus, by Theorem $7, K \in U E C_{\Delta}$. By the compactness theorem for arithmetical classes, we see that the subscript $\Delta$ may be removed from the above remark. We now proceed to the main result on unions of chains.

For our subsequent discussion we introduce the following notation. Let $\mathfrak{R} \in S^{*}(\mathfrak{B})$ and let $\Sigma$ be the set of all sentences constructed with the constants $c_{a}$ for $a \in A$. We consider a new set of sentences $\Sigma(\mathfrak{A}, \mathfrak{B})$ with constants $c_{b}$ for $b \in B$ consisting of :

(1) the set of all sentences of $\Sigma$ which hold in $\mathfrak{A}$ under the interpretation of $a$ for $c_{a}$, for all $a \in A$, and

(2) the description of $\mathfrak{B}$.

We are now ready to prove the following lemmas.

Lemma 8. $\Sigma(\mathfrak{A}, \mathfrak{B})$ has a model.

Proof. Any finite subset $\Sigma^{\prime}$ of $\Sigma(\mathfrak{A}, \mathfrak{B})$ will contain only a finite number of constants $c_{b}$ for $b \in B$. Let $B^{\prime}$ be the finite subset of $B$ determined by the constants occurring in $\Sigma^{\prime}$ and let $\mathfrak{B}^{\prime}$ be the corresponding subsystem. By Definition 5, there exists a subsystem $\mathfrak{A}^{\prime}$ of $\mathfrak{A}$ which is isomorphic to $\mathfrak{B}^{\prime}$ and such that the isomorphism $f$ is the identity mapping on the set $B^{\prime} \cap A$. Clearly, $\mathfrak{A}$ is a model for $\Sigma^{\prime}$ if each constant $c_{b}$ for $b \in B^{\prime}$ is interpreted as the element $f(b) \in A$. Thus, by the compactness theorem, $\Sigma(\mathfrak{A}, \mathfrak{B})$ has a model.

Lemma 9. Let $K \in A C_{\Delta}, \mathfrak{\mho}_{0} \in K, \mathfrak{A}_{0} \in S^{*}\left(\mathfrak{\Im}_{0}\right)$, and $\Sigma_{0}=\Sigma\left(\mathfrak{A}_{0}, \mathfrak{\Im}_{0}\right)$. Then there exist an infinite sequence of models $\mathfrak{A}_{0}, \mathfrak{S}_{0}, \mathfrak{A}_{1}, \mathfrak{E}_{1}, \cdots$, $\mathfrak{A}_{n}, \mathfrak{E}_{n}, \cdots$ and an infinite sequence of sets of sentences $\Sigma_{0}, \Sigma_{1}, \cdots$, $\Sigma_{n}, \ldots$ such that for each natural number $n$ the following hold:

(i) $\mathfrak{A}_{n} \in S^{*}\left(\mathfrak{E}_{n}\right)$.

(ii) $\mathfrak{\complement}_{n} \in S\left(\mathfrak{A}_{n+1}\right)$ and $\mathfrak{E}_{n} \in K$.

(iii) $\Sigma_{n}=\Sigma\left(\mathfrak{A}_{n}, \mathfrak{S}_{n}\right)$.

(iv) Every sentence of $\Sigma_{n}$ holds in $\mathfrak{A}_{n+1}$.

(v) If $m \leqq n$, then $\mathfrak{A}_{n}$ is an arithmetical extension of $\mathfrak{A}_{m} \cdot{ }^{4}$

Proof. The lemma is proved by mathematical induction. Assume we already have the models $\mathfrak{A}_{0}, \mathfrak{C}_{0}, \cdots, \mathfrak{A}_{n}, \mathfrak{C}_{n}$, and the sets of

${ }^{3}$ This result was known to Tarski and was orally communicated to the author.

${ }^{4}$ For the notion of arithmetical extensions, cf. Definition 1.6 in [9]. 
sentences $\Sigma_{0}, \cdots, \Sigma_{n}$. We can now obtain the model $\mathfrak{A}_{n+1}$ with the aid of Lemma 8 , the inductive hypothesis, and the fact that $K$ is closed under taking isomorphic models. In order to prove (v), by Theorem 1.8(ii) of [9] it is sufficient to show that $\mathfrak{A}_{n+1}$ is an arithmetical extension of $\mathfrak{A}_{n}$; i.e., that

(1) every sentence constructed with the constants $c_{a}$, for $a \in A_{n}$, which holds in $\mathfrak{A}_{n}$ also holds in $\mathfrak{A}_{n+1}$.

Condition (1), of course, follows immediately from (iii) and (iv). From (v) and Corollary 1.7 of [9], we see that $\mathfrak{A}_{0}$ and $\mathfrak{A}_{n+1}$ are arithmetically equivalent. Since $\mathfrak{A}_{0} \in S^{*}(K)$ and $S^{*}(K) \in U E C_{\Delta}$, therefore $\mathfrak{A}_{n+1} \in S^{*}(K)$. We now simply let $\mathfrak{S}_{n+1}$ be such a model that $\mathfrak{E}_{n+1} \in K$ and $\mathfrak{A}_{n+1} \in S^{*}\left(\mathfrak{S}_{n+1}\right)$. Obviously, $\mathfrak{E}_{n} \in S\left(\mathfrak{A}_{n+1}\right)$. The induction is complete and the lemma is proved.

Lemma 10. Given the sequence of models as in Lemma 9, $\mathfrak{A}_{0}$ and $\cup \mathfrak{A}_{n}$ are arithmetically equivalent.

Proof. By Theorem 1.9 of [9], $\cup \mathfrak{A}_{n}$ is an arithmetical extension of $\mathfrak{A}_{0}$. Thus, by Corollary 1.7 of [9], the conclusion of the lemma follows.

Given a class $K$ we let $C L(K)=\{\mathfrak{A} ;$; there exists a $\mathfrak{B} \in K$ such that $\mathfrak{A}$ is arithmetically equivalent with $\mathfrak{B}\}$.

Theorem 11. If $K \in A C_{\Delta}$, then $S^{*}(K)=C L(\mathrm{U}(K))$.

Proof. In order to prove the inclusion $C L(\mathrm{U}(K)) \subseteq S^{*}(K)$ it is sufficient, by Theorem 7 , to prove that $\mathrm{U}(K) \subseteq S^{*}(K)$. To this end, let $\mathfrak{A}$ be the union of a chain of models $\mathfrak{A}_{i} \in K$, with $i \in I$. We consider the following set of sentences $\Sigma^{\prime}$ consisting of:

(1) The set $\Sigma$ of sentences characterizing $K$.

(2) The description of $\mathfrak{A}$.

(3) For each finite subset $\left\{a_{1}, \cdots, a_{n}\right\}$ of $A$ and each natural number $m$, the sentence $\nu\left(c_{a_{1}}, \cdots, c_{a_{n}}\right)$ introduced in $(* *)$.

Any finite subset $\Sigma^{\prime \prime}$ of $\Sigma^{\prime}$ will contain only a finite number of constants $c_{a}$ for $a \in A$. Any finite number of elements of $A$ belongs to $A_{i}$ for some $i \in I$. It is obvious that $\mathfrak{A}_{i}$ will be a model for $\Sigma^{\prime \prime}$. Thus, by the compactness theorem, there exists a model $\mathfrak{B}$ for $\Sigma^{\prime}$. We see from the construction of $\Sigma^{\prime}$ that $\mathfrak{B} \in K$ and that there exists a model $\mathfrak{A}^{\prime}$ such that $\mathfrak{H}^{\prime} \in S^{*}(\mathfrak{B})$ and $\mathfrak{A}$ isomorphic to $\mathfrak{A}^{\prime}$. Thus $\mathfrak{A} \in S^{*}(K)$.

In order to prove the inclusion $S^{*}(K) \subseteq C L(\mathrm{U}(K))$, let $\mathfrak{A} \in S^{*}(\mathfrak{E})$ and $\mathfrak{S} \in K$. Letting $\mathfrak{A}=\mathfrak{A}_{0}$ and $\mathfrak{S}=\mathfrak{S}_{0}$, we repeat the construction of the sequences $\mathfrak{A}_{0}, \mathfrak{S}_{0}, \ldots, \mathfrak{A}_{n}, \mathfrak{S}_{n}, \cdots$ and the sets $\Sigma_{0}, \ldots$, $\Sigma_{n}, \cdots$ as in Lemma 9. It is clear that $U \mathfrak{A}_{n}=U \mathfrak{S}_{n}$. Since $\mathfrak{夭}_{n} \in K$ for each $n, \cup \mathfrak{S}_{n} \in \mathrm{U}(K)$ and hence $U \mathfrak{A}_{n} \in \mathrm{U}(K)$. By Lemma 10, $\mathfrak{A}$ and 
$\cup \mathfrak{A}_{n}$ are arithmetically equivalent, thus $\mathfrak{A} \in C L(\bigcup(K))$. The theorem is proved.

Theorem 12. If $K \in A C_{\Delta}$, then $K \in U E C_{\Delta}$ if, and only if, $\mathrm{U}(K) \subseteq K$.

Proof. Assume $K \in U E C_{\Delta}$. By Theorem $6, S^{*}(K) \subseteq K$ and, by Theorem 11, $\mathrm{U}(K) \subseteq K$. On the other hand, if $\mathrm{U}(K) \subseteq K$, then, by Theorem 11, $C L(\mathrm{U}(K))=C L(K)=K=S^{*}(K)$, thus $S^{*}(K) \subseteq K$. By Theorem 6 again, $K \in U E C_{\Delta}$. The theorem is proved.

It is now of some interest to ask whether $K \in A C_{\Delta}$ implies $\mathrm{U}(K)$ $\in U E C_{\Delta}$. In what follows we exhibit a $K \in A C$ such that $\mathrm{U}(K)$ $\notin U E C_{\Delta}$.

We consider relational systems $\langle A, R\rangle$ formed by a nonempty set $A$ and a binary relation $R$ over $A$. Let $K$ be the arithmetical class of models $\langle A, R\rangle$ determined by the conditions: the relation $R$ is a simply ordering relation on the elements of $A$, if an element of $A$ has an immediate predecessor then it also has an immediate successor, and there exists an element of $A$ which is the successor of every element of $A$. Let ordinals be defined in such a way that each ordinal is the set of all smaller ordinals, and let $\leqq$ be the well-ordering relation among ordinals. If we let $\omega$ denote the least infinite ordinal, then the following will hold:

$$
\langle\omega, \leqq) \in S^{*}(\langle\omega+1, \leqq\rangle), \quad\langle\omega+1, \leqq\rangle \in K,
$$

and

$$
\langle\omega, \leqq\rangle \in S^{*}(K) .
$$

Since no finite subsystem of $\langle\omega$, $\leqq$ with more than one element belongs to $K$, and no infinite subsystem of $\langle\omega, \leqq\rangle$ belongs to $K$, we see that $\langle\omega, \leqq\rangle \notin \mathrm{U}(K)$. If $\mathrm{U}(K) \in U E C_{\Delta}$, then by Theorem $11, S^{*}(K)$ $=\mathrm{U}(K)$. Thus $\langle\omega, \leqq\rangle \in \mathrm{U}(K)$ which is a contradiction.

In the concluding part of the paper we apply our results to those arithmetical classes which are convex. ${ }^{5}$

Definition 13. $K \in A C_{\Delta}$ is convex if, and only if, whenever $\mathfrak{A}, \mathfrak{B}$, $\mathfrak{C}$ $\in K, \mathfrak{B}, \mathfrak{S} \in S(\mathfrak{A})$, and $\mathfrak{B} \cap \mathfrak{C}$ is not empty, then $\mathfrak{B} \cap \mathfrak{S} \in K$.

Theorem 14. If $K \in A C_{\Delta}$ and $K$ is convex, then $U(K) \subseteq K$.

Proof. Let $\mathfrak{A}_{i}, i \in I$, be a chain of models of $K$, and let $\mathfrak{A}=U \mathfrak{A}_{i}$. By

5 The definition of a "convex algebra of axioms" is found in Robinson [6]. Stated in terms of arithmetical classes, his notion is much stronger than what we have assumed here. Thus, our Theorem 14 is essentially a strengthening of (9.1.3) of [6]. Theorem 14 was announced by the author in [1]; we point out here that all other results announced in [1] can be proved in a manner similar to the proof of Theorem 14. 
the compactness theorem and the fact that $K \in A C_{\Delta}$, we see that there exists a model $\mathfrak{B} \in K$ such that $\mathfrak{A} \in S(\mathfrak{B})$. Let us now in troduce constants $c_{b}$ for each $b \in B$ and an unary predicate symbol $P$. We consider the set of sentences $\Sigma^{\prime}$ consisting of:

(1) The set $\Sigma$ of sentences characterizing $K$.

(2) The set $\Sigma^{(P)}$ obtained by relativizing all sentences of $\Sigma$ to $P .^{\circ}$

(3) The set of all sentences $P\left(c_{a}\right)$ for all $a \in A$.

(4) The set of all sentences $\sim P\left(c_{b}\right)$ for all $b \in B-A$.

(5) The description of $\mathfrak{B}$.

Every finite subset $\Sigma^{\prime \prime}$ of $\Sigma^{\prime}$ will involve at most a finite number of constants. For some $\mathfrak{A}_{j}$, the set $A_{j}$ will contain all those elements corresponding to those constants in $\Sigma^{\prime \prime}$. Clearly, $\mathfrak{B}$ will be a model for $\Sigma^{\prime \prime}$ if the unary predicate is interpreted as the set $A_{j}$. Hence, by the compactness theorem, there exists a model $\subseteq$ for $\Sigma^{\prime}$. Let $D$ be the set of elements of $C$ which is the interpretation of $P$ in $C$, and let $D$ be the corresponding subsystem of $\mathfrak{C}$. By (2), $\mathfrak{D} \in K$. Let $A^{\prime}$ be the set of elements of $C$ which are interpretations of the constants $c_{a}$, for $a \in A$, and let $\mathfrak{H}^{\prime}$ be the corresponding subsystem. Similarly, let $B^{\prime}$ be the set of elements of $C$ which are interpretations of the constants $c_{b}$, for $b \in B$, and let $\mathfrak{B}^{\prime}$ be the corresponding subsystem. We see that $\mathfrak{X} \cong \mathfrak{Y}^{\prime}, \mathfrak{B} \cong \mathfrak{B}^{\prime}$, and $\mathfrak{B}^{\prime} \cap \mathfrak{D}=\mathfrak{H}^{\prime}$. Thus $\mathfrak{H}^{\prime} \in K$, and, finally, $\mathfrak{A} \in K$. The theorem is proved.

Corollary 15. If $K \in A C_{\Delta}$ and $K$ is convex, then $K \in U E C_{\Delta}$.

\section{REFERENCES}

1. C. C. Chang, A remark on convex classes, Bull. Amer. Math. Soc. Abstract 60-4-579.

2. - On the union of chains of models, Bull. Amer. Math. Soc. Abstract 62-6-775.

3. K. Gödel, Die Vollständigkeit der Axiome des logischen Funktionenkalküls, Monatshefte für Mathematik und Physik, vol. 37 (1930) pp. 349-360.

4. J. Los and R. Suszko. On the infinite sums of models, Bull. Acad. Polon. Sci. Cl. III vol. 3 (1955) pp. 201-202.

5. - On the extending of models, IV, Fund. Math. vol. 44 (1957) pp. 52-60.

6. A. Robinson, On the metamathematics of algebra, Amsterdam, 1951.

7. A. Tarski, Contributions to the theory of models, Parts I and II, Indag. Math. vol. 16 (1954) pp. 572-588.

8. A. Tarski, A. Mostowski, and R. M. Robinson, Undecidable theories, Amsterdam, 1953.

9. A. Tarski and R. L. Vaught, Arithmetical extensions of relational systems, Compositio Math. vol. 13 (1957) pp. 81-102.

University of California, Los Angeles

- For the notion of relativizing a sentence to an unary predicate $P$, cf. $[8$, pp. $24-$ $25]$. 